\title{
Factors associated with willingness to participate in a heroin prescription program among injection drug users
}

\author{
Cari L. Miller, MSc \\ Steffanie A. Strathdee, $\mathrm{PhD}$ \\ Thomas Kerr, PhD \\ Will Small, MA \\ Kathy Li, MSc \\ Evan Wood, PhD
}

\section{ABSTRACT}

Randomized controlled trials of prescription heroin have shown success in reducing drug-related harm among chronic opiate injection drug users (IDUs) in several European nations. We sought to explore willingness to participate in a heroin trial among a well-characterized North American cohort of IDUs, and therefore performed analyses of factors associated with willingness to participate in a prescription heroin trial among IDUs enrolled in the Vancouver Injecting Drug Users Study (VIDUS). Of 410 current heroin injectors followed between May and November 2002, injecting heroin frequently (more than once daily) lodds ratio (OR) 1.33; 95 percent confidence interval (CI) 1.06 to 1.69] and being enrolled in methadone maintenance therapy (MMT; OR 1.33, 95 percent CI 1.06 to 1.69) were associated with willingness to participate in a trial. In subanalyses, statistical associations with willingness to participate in a trial among current MMT users were frequent injection of heroin (OR 2.12, CI 1.16 to 3.88) and speedballs (OR 2.57, CI 1.02 to 6.48), frequent crack cocaine use (OR 1.84, CI 1.11 to 3.06), lending of syringes (OR 3.22, CI 1.08 to 9.65), and requiring help to inject (OR 1.83, CI 1.01 to 3.33). Among IDUs, willingness to enroll in a heroin prescription program was associated with highintensity heroin injection and high-risk behaviors and was particularly prevalent among individuals who have been unable to significantly reduce their injection drug use on MMT alone. These findings indicate that a clinical trial of prescribed heroin should be able to enroll an appropriate sample of drug users and properly assess the treatment potential of prescribed opiate pharmacotherapy.

Key words: prescription heroin, methadone maintenance therapy, injection drug use, treatment

\section{INTRODUCTION}

Clinical controlled trials of prescription heroin in Europe have shown success in reducing drug-related harm among chronic opiate injection drug users (IDUs) while showing no elevated health risks or evidence of having increased the number of IDUs., ${ }^{1,2}$ The findings from these studies have prompted the expansion of existing heroin prescription programs and the addition of new trials. ${ }^{3,4}$ Despite the rapid spread of human immunodeficiency virus (HIV) infection that has occurred in cities across North America, the effects of heroin prescription remain unknown in this setting. ${ }^{5,6}$ However, Canadian investigators have developed a protocol to test the feasibility of a prescription heroin program in North America through the establishment of several clinical control trial sites in cities with a high prevalence of IDUs. ${ }^{7}$ The Vancouver, British Columbia prescription heroin trial began in the spring of 2005 .

Although heroin prescription programs are being considered in other settings, we are aware of no study investigating willingness among IDUs to enroll in such programs. We therefore undertook this study to investigate the acceptability of prescription heroin among a wellcharacterized cohort of IDUs at the Vancouver trial site.

\section{METHODS AND MATERIALS}

We investigated correlations with willingness to participate in a heroin prescription trial among IDUs enrolled in the Vancouver Injection Drug User Study (VIDUS), a prospective study of IDUs who have been recruited through self-referral and street outreach from Vancouver's Downtown Eastside since May 1996. To date, over 1,500 participants have been enrolled. The cohort has been described in detail previously., 5 Ethical 
approval for the project was provided by the University of British Columbia's Ethics Committee on Human Experimentation.

The current analyses were restricted to VIDUS participants who were current heroin injectors at the time of interview and who were followed between May 2002 and November 2002 and replied to the following question asked of participants during this period: "If a structured prescription heroin program similar to methadone was available, would you use it?" We used contingency table analysis to compare sociodemographic and risk variables between participants who were willing and those unwilling to participate in a prescription heroin trial.

For the current analyses, variables of interest included baseline sociodemographics of gender and ethnicity (aboriginal vs. other), as well as continuous variables referring to the previous six months at the time of interview, including unstable housing, heroin, cocaine and speedball injection, crack cocaine smoking, sex-trade involvement, binge use of drugs, requiring help to inject, public injecting, and syringe borrowing and lending. To be consistent with our previous work, unstable housing was defined as living arrangements that included singleroom occupancy hotels and homelessness, and sex-trade involvement was measured as having sexual partners with whom sex was traded for money, drugs, or shelter. "Borrowing" refers to the use of used syringes, and "lending" refers to lending used syringes. "Speedball" refers to a mixture of heroin and cocaine. Binge drug use is well understood by local IDUs and generally refers to compulsive high-intensity injection drug use that differs from normal patterns of consumption, often very frequent cocaine and/or polydrug injection for periods ranging from a few days to a few weeks. Requiring help with injecting is a common practice among many IDUs whereby others are relied on to inject the drugs due to collapsed veins and/or difficulty accessing veins. All continuous variables were elicited in reference to the six months preceding the interview.

\section{RESULTS}

Of 410 IDUs who met eligibility criteria, 256 (62 percent) reported willingness to participate in a prescription heroin program. Willingness was associated with sex-trade involvement (OR 1.88, 95 percent CI 1.08 to 3.27), frequent (more than once daily) heroin (OR 2.89, 95 percent CI 1.90 to 4.40) and speedball injection (OR 2.13, 95 percent CI 1.13 to 4.03), and current use of methadone maintenance therapy (MMT) (OR 1.77, 95 percent CI 1.18 to 2.65 ).

We were aware that a substantial proportion of opiate addicts in the cohort may have been excluded from the study because they were currently on MMT and not actively injecting heroin. We therefore performed subanalyses of the 301 individuals who were followed between May 2002 and November 2002 and who reported being currently on MMT. Willingness was associated with lending used syringes (OR 3.22, 95 percent CI 1.08 to 9.65), requiring help to inject (OR 1.83, 95 percent CI 1.01 to 3.33), and frequent heroin (OR 2.12, 95 percent CI 1.16 to 3.88), crack (OR 1.84, 95 percent CI 1.11 to 3.06), and speedball use (OR 2.57, 95 percent CI 1.02 to 6.48).

\section{DISCUSSION}

Vancouver, British Columbia, has been the site of an explosive outbreak of HIV infection among IDUs. To respond to the growing public health concerns in this population, MMT services have been revised and expanded in recent years to increase the availability of MMT to Vancouver-area IDUs. MMT in this setting is available free of charge, prescribed primarily by community physicians, and dispensed by community pharmacists. Average daily dosages range from 75 to $80 \mathrm{mg}$, and while efforts have been made to expand the program, waitlists still exist. Currently, there are no regulatory requirements to discharge MMT patients for infractions, including ongoing illicit drug use. ${ }^{9}$

Our finding of an association between current MMT users, ongoing heroin injection, and willingness to participate in a heroin trial suggests that MMT may not be a sufficient treatment for some heroin injectors and corroborates similar findings in other studies of IDUs. ${ }^{1,10,11}$ Prescription heroin and heroin combined with MMT in other trial settings has been found to be more effective than MMT on its own in reducing continued frequent injection, as well as illegal activities and cocaine use. ${ }^{1,12}$ The types of potential benefits that could occur in this high-risk population include a reduction in drug-related harm and blood-borne infections.

As would be expected, participants that were willing to participate in a heroin trial were those with uncontrolled addictions and who were injecting heroin and speedballs on a frequent basis, often despite the use of MMT. This finding underscores the fact that many IDUs, particularly high-intensity polydrug users, are willing to engage in therapeutic treatment programs to gain control over or change their addictive behavior. Sex-trade involvement, requiring help to inject, and frequent crack and speedball use in this setting have been associated with a number of high-risk practices, including syringe borrowing and lending.

In summary, willingness to enroll in a heroin prescription program was associated with high-intensity heroin and cocaine injection, sex-trade involvement, and use of MMT. Among individuals currently on MMT, willingness was associated with ongoing high-risk behaviors that have been associated with HIV incidence, criminal activity, and community harm. These findings provide evidence that the initiation of a heroin prescription trial may 
be associated with substantial public health and community benefits and support the rationale for heroin prescription in Vancouver and potentially other settings in North America.

\section{ACKNOWLEDGMENTS}

The authors would particularly like to thank the VIDUS participants for their willingness to participate in the study. Cari Miller is supported by a Canadian Graduate Scholarship and by the Michael Smith Foundation for Health Research (MSFHR). We also thank Bonnie Devlin, John Charette, Caitlin Johnston, Robin Brooks, Steve Kain, Dave Isham, Will Small, Peter Vann, and Vanessa Volkommer for their research and administrative assistance. This study would not have been possible without the financial support of Status of Women Canada. This study was further supported by Canadian Institutes of Health Research grant 122258 .

Cari L. Miller, MSc, British Columbia Centre for Excellence in HIV/AIDS, St. Paul's Hospital, Vancouver, British Columbia, Canada.

Steffanie A. Strathdee, PhD, Division of International and Cross-Cultural Medicine, University of California, San Diego, San Diego, California.

Thomas Kerr, PhD, British Columbia Centre for Excellence in HIV/AIDS, St. Paul's Hospital, and Department of Medicine, University of British Columbia, Vancouver, British Columbia, Canada.

Will Small, MA, British Columbia Centre for Excellence in HIV/ AIDS, St. Paul's Hospital, Vancouver, British Columbia, Canada. Kathy Li, MSc, British Columbia Centre for Excellence in HIV/ AIDS, St. Paul's Hospital, Vancouver, British Columbia, Canada. Evan Wood, PhD, Division of Epidemiology and Population Health, British Columbia Centre for Excellence in HIV/AIDS, St. Paul's Hospital, and Department of Medicine, University of British Columbia, Vancouver, British Columbia, Canada.

\section{REFERENCES}

1. van den Brink W, Hendriks VM, Blanken P, et al.: Medical prescription of heroin to treatment resistant heroin addicts: Two randomised controlled trials. BMJ. 2003; 327(7410): 310.

2. Rehm J, Gschwend P, Steffen T, et al.: Feasibility, safety, and efficacy of injectable heroin prescription for refractory opioid addicts: A follow-up study. Lancet. 2001; 358(9291): 1417-1423. 3. Hall W: The contribution of research to Australian policy responses to heroin dependence 1990-2001: A personal retrospection. Addiction. 2004; 99(8): 1063-1064.

4. Brissette S: Medical prescription of heroin-A review. Can HIV AIDS Policy Law Rev. 2001; 6(1-2): 1, 92-98.

5. Strathdee SA, Patrick DM, Currie SL, et al.: Needle exchange is not enough: Lessons from the Vancouver injecting drug use study. AIDS. 1997; 11(8): F59-F65.

6. Des Jarlais DC, Friedman SR: HIV epidemiology and interventions among injecting drug users. Int J STD AIDS. 1996; 7(Suppl 2): $57-61$.

7. Schechter M: NAOMI-Her time has come. North American Opiate Medication Initiative. J Urban Health. 2002; 79(2): 164165.

8. Tyndall MW, Craib KJ, Currie S, et al.: Impact of HIV infection on mortality in a cohort of injection drug users. J Acquir Immune Defic Syn. 2001; 28(4): 351-357.

9. Anderson J, Warren L: Client retention in the British Columbia Methadone Program, 1996-1999. Can J Pub Health. 2004; 95: 104-109.

10. Bammer G, Dobler-Mikola A, Fleming PM, et al.: The heroin prescribing debate: Integrating science and politics. Science. 1999; 284(5418): 1277-1278.

11. Gibson DR, Flynn NM, McCarthy JJ: Effectiveness of methadone treatment in reducing HIV risk behavior and HIV seroconversion among injecting drug users [Editorial]. AIDS. 1999; 13(14): 1807-1818.

12. Steffen T, Christen S, Blattler R, et al.: Infectious diseases and public health: risk-taking behavior during participation in the Swiss program for a medical prescription of narcotics (PROVE). Subst Use Misuse. 2001; 36(1-2): 71-89. 